Article

\title{
Biocompatibility of Poly(ester amide) (PEA) Microfibrils in Ocular Tissues
}

\author{
Martina Kropp ${ }^{1}$, Katharina-Marie Morawa ${ }^{2}$, George Mihov ${ }^{4}$, Anna Katharina Salz ${ }^{3}$, \\ Nina Harmening ${ }^{1}$, Astrid Franken ${ }^{4}$, Anja Kemp ${ }^{4}$, Aylvin A. Dias ${ }^{4}$, Jens Thies ${ }^{4}$, \\ Sandra Johnen ${ }^{3}$, Gabriele Thumann ${ }^{1,3, *}$
}

1 Department of Vitreoretinal Surgery, University Hospitals of Geneva, Rue Alcide-Jentzer 22, 1205 Geneva, Switzerland; E-Mails: martina.kropp@hcuge.ch (M.K.); nina.harmening@hcuge.ch (N.H.) Department of Ophthalmology, University Hospital Rheinisch-Westfälische Technische Hochschule (RWTH) Aachen, Pauwelsstrasse 30, 52074 Aachen, Germany; E-Mail: katharina.morawa@rwth-aachen.de

3 Interdisziplinäres Zentrum für Klinische Forschung (IZKF) Aachen, University Hospital Rheinisch-Westfälische Technische Hochschule (RWTH) Aachen, Pauwelsstrasse 30, 52074 Aachen, Germany; E-Mails: a.salz@gmx.de (A.K.S.); sjohnen@ukaachen.de (S.J.)

4 DSM, Urmonderbaan 22, Geleen 6167 RD, The Netherlands;

E-Mails: george.mihov@dsm.com (G.M.); astrid.franken@dsm.com (A.F.); anja.kemp@dsm.com (A.K.); aylvin.dias@dsm.com (A.A.D.); jens.thies@dsm.com (J.T.)

* Author to whom correspondence should be addressed; E-Mail: gabriele.thumann@unige.ch; Tel.: +41-22-382-8394; Fax: +41-22-382-8421.

Received: 30 November 2013; in revised form: 10 January 2014 / Accepted: 13 January 2014 / Published: 21 January 2014

Abstract: Drug delivery systems (DDS) are able to deliver, over long periods of time, therapeutic concentrations of drugs requiring frequent administration. Two classes of DDS are available, biodegradable and non-biodegradable. The larger non-biodegradable implants ensure long-term delivery, but require surgical interventions. Biodegradable biomaterials are smaller, injectable implants, but degrade hydrolytically and release drugs in non-zero order kinetics, which is inefficient for long-term sustained drug release. Biodegradable poly(ester amides) (PEAs) may overcome these difficulties. To assess their ocular biocompatibility and long-term behavior, PEA fibrils were analyzed in vitro and in vivo. In vitro, incubation in vitreous humor changes to PEA structure, suggests degradation by surface erosion, enabling drug release with zero order kinetics. Clinical and histological analysis of PEA fibrils implanted subconjunctivally and intravitreally showed 
the absence of an inflammatory response or other pathological tissue alteration. This study shows that PEA fibrils are biocompatible with ocular environment and degrade by surface erosion.

Keywords: biomaterials; biodegradation; drug delivery; controlled drug release; amino acid

\section{Introduction}

Ocular drug delivery is a rapidly expanding pharmaceutical market and a key target area for the pharmaceutical industry. An unmet need in ocular drug delivery is the sustained, zero order delivery of drugs and other bioactive molecules to the posterior segment of the eye [1]. Successful ophthalmic therapy requires that an effective concentration reaches the specific target tissue. This is particularly difficult when the target tissue is the posterior segment, since, when applied to the cornea, most drugs do not penetrate in sufficient quantity to the posterior segment [2]. Treatment of posterior segment disorders often requires the administration of drugs over extended periods of time, which is usually achieved by injection of the drug as close to the diseased tissue as possible. For exudative age-related macular degeneration (AMD), choroidal neovascularization is controlled by monthly intravitreal injections of anti-VEGF (vascular endothelial growth factor) throughout the life of the patient, with substantial risk of side effects including endophthalmitis and ocular hypertension [3-6].

To avoid the complications associated with frequent intraocular injections, a sustained-release drug delivery device would be required. Sustained intraocular drug delivery systems (DDS), that allow the long-term release of the active substance with the same therapeutic effect as multiple injections, are available, but have not proven satisfactory. One of the first non-degradable intraocular sustained drug delivery biomaterials, Vitrasert ${ }^{\circledR}$ (Bausch \& Lomb), was approved by the FDA (U.S. Food and Drug Administration) and introduced to the market in 1996 for the treatment of cytomegalovirus (CMV) retinitis in patients infected with human immunodeficiency virus [2,7]. Vitrasert ${ }^{\circledR}$ releases the antiviral drug ganciclovir over five to eight months. However, a number of complications associated with non-biodegradable materials, such as surgical removal of depleted inserts, visual acuity loss, vitreous humor hemorrhage, retinal detachment, vitreous humor detachment, vitreous humor traction, hypotony, severe post-operative inflammation, etc. and non-compatibility with protein drugs have prevented the wide use of non-biodegradable materials for intraocular therapy [8-10].

Currently, micro- and nanoparticles prepared from linear, biodegradable polyesters such as poly(lactic-co-glycolic acid) (PLGA) are used [11] and in 2009 an injectable PLGA intravitreal insert, OZURDEX $^{\circledR}$, was approved by the FDA and is used successfully for the treatment of macular edema by releasing dexamethasone over a period of about three months [2,12]. PLGA is not used widely for intraocular drug delivery, in part because the kinetics of degradation and drug release are irregular. There is an initial burst of drug release, followed by a phase of sustained release and a final phase in which all remaining drug is set free $[1,13]$. In addition to the asymmetrical release of drug, PLGA is hydrolyzed into lactic acid and glycolic acid, which transiently decrease vitreous humor to $\mathrm{pH} 7$ and increases the risk of inflammation before being metabolized [12,14]. In addition, PLGA has been reported to show low compatibility with acid sensitive bio-pharmaceuticals [14,15]. 
Ideally a DDS should be compatible with all or most drugs and its degradation should enable a zero-order drug release for efficient treatment. Degradation of the DDS should occur over an extended period of time to provide the patient effective treatment. A new class of amino acid based poly(ester amide) (PEA) polymers have been developed that meet the requirements of an efficient and useful drug delivery system [16-20].

PEAs are synthetic polycondensation products consisting of biocompatible building blocks such as hydrophobic L-amino acids, aliphatic di-carboxylic acids and $\alpha, \omega$-diols. The use of amino-acids as components of the PEA polymers confers several advantages. Degradation in vivo is accomplished by enzymes normally present in tissues, degradation begins at the surface allowing controlled zero order degradation [21,22], drugs are released with a zero order kinetics and the incorporation of amino acid-based building blocks [20] provide one or more functional groups along the polymer chain allowing modification of the polymer to tailor its physical and chemical properties, bio-erosion and performance as drug releasing vehicles, e.g. [16]. Recently, this class of polymers have been shown to be compatible with the cardiovascular environment $[18,19]$ and successfully applied as drug eluting coating on coronary stents [18].

However, most of the work done on PEA polymers has been limited to in vitro analysis and rare in vivo works investigations to coat cardiovascular stents. To our knowledge, solid PEA fibers have not been examined as an implantable drug delivery system. The ability to encapsulate and release a variety of drug species, including protein drugs, coupled with the lack of side effects of degradation products and versatile processability of the PEA polymers to be manufactured as injectable fibrils and microparticles, makes PEA platform ideal as a vehicle for the prolonged delivery of ophthalmic drugs.

Here we report the development of PEA microfibrils as ocular implants, and shown that the studied PEA polymer can be effectively extruded to injectable microfibrils with therapeutic potential for ophthalmology. Furthermore, PEA microfibrils were implanted in rabbits subconjunctivally and intravitreally by injection and shown to be well tolerated for the studied period up to eight weeks triggering only healthy inflammatory responses. The fibrils degrade by surface erosion in presence of proteases which makes them attractive for further research as a potential long-term drug eluting device.

\section{Experimental Section}

\subsection{Polymer Preparation}

Unless specified otherwise, all chemicals were purchased from Sigma-Aldrich (St. Louis, MI, USA). ${ }^{1} \mathrm{H}$ NMR analysis was performed on a Varian Inova 300 spectrometer (Varian Inc./Agilent Technologies, Santa Clara, CA, USA) using a $10 \mathrm{mg} / \mathrm{mL}$ polymer solution in deuterated chloroform. Differential scanning calorimetry (DSC) was performed using a Mettler Toledo 822e DSC (Mettler Toledo, Greifensee, Switzerland) connected to an Intercooler and an auto robot TS0801RO (Mettler Toledo, Greifensee, Switzerland).

Polymer molecular weight and polydispersity were evaluated by means of gel permeation chromatography (GPC) on Waters separations module with column heater type e2695 (Waters, Milford, CT, USA) and Waters RI detector type 2414 (Waters). A styragel HR5E and styragel HR2 with a guard column at $50{ }^{\circ} \mathrm{C}$ were used (Waters). As an eluent tetrahydrofuran (THF) with a flow rate 
of $1.0 \mathrm{~mL} / \mathrm{min}$ and an injection volume of $100 \mu \mathrm{L}$ was applied. Calculations were done relatively to polystyrene calibration standard.

The polymer used in this study was synthesized following the procedure reported by Katsarava [23]. Briefly, the polymer was prepared via polycondensation of 0.45 equivalents of di-p-toluenesulfonic acid salts of bis-(L-leucine)1,4-dianhydro sorbitol diester (1), 0.30 equivalents of bis-(L-leucine) $\alpha, \omega$-hexane diol-diester (2), 0.25 equivalents of lysine benzyl-ester (3), and one equivalent of di-N-hydroxysuccinimide ester of sebacic acid (4) in anhydrous DMSO and triethylamine added in a glass vessel with overhead stirrer under a nitrogen atmosphere (Figure 1). The usage of pre-activated acid in the reaction allows polymerization at relative low temperature $\left(65^{\circ} \mathrm{C} ; 48 \mathrm{~h}\right)$ resulting in side-products-free polycondensate and predictable degradation products. The polymer was isolated from the reaction mixture in two precipitation steps resulting in a white amorphous material of $54 \mathrm{kDa}$ average number molecular weight $(\mathrm{Mn})$. The relative ratio of the different building blocks in the polymer was calculated from the ${ }^{1} \mathrm{H}$ NMR spectrum. The integration of characteristic protons signals for 1,6-hexanediol (m, 4.1-3.9 ppm), lysine (m, 3.0-2.9 ppm) and 1,4:3,6-dianhydro-D-sorbitol (s, 4.7 ppm) was used to calculate the relative molar ratio of the amino acid containing building blocks (Figure 2). The results are shown in Table 1 reveal that actual copolymer composition is very close to the theoretical one. To obtain a uniform mixture, the polymer was cryomilled in Retsch ZM200 (Retsch, Haan, Germany) equipment in the presence of $0.25 \% \mathrm{w} / \mathrm{w}$ fluorescein octadecyl ester and $0.25 \% \mathrm{w} / \mathrm{w}$ ascorbic acid as an antioxidant.

Figure 1. The poly(ester amide) (PEA) polymer is synthesized from the four monomers: bis-(L-leucine) 1,4-dianhydro sorbitol diester (1), bis-(L-leucine) $\alpha, \omega$-hexane diol-diester (2), lysine benzyl-ester (3), and di-N-hydroxysuccinimide ester of sebacic acid (4).

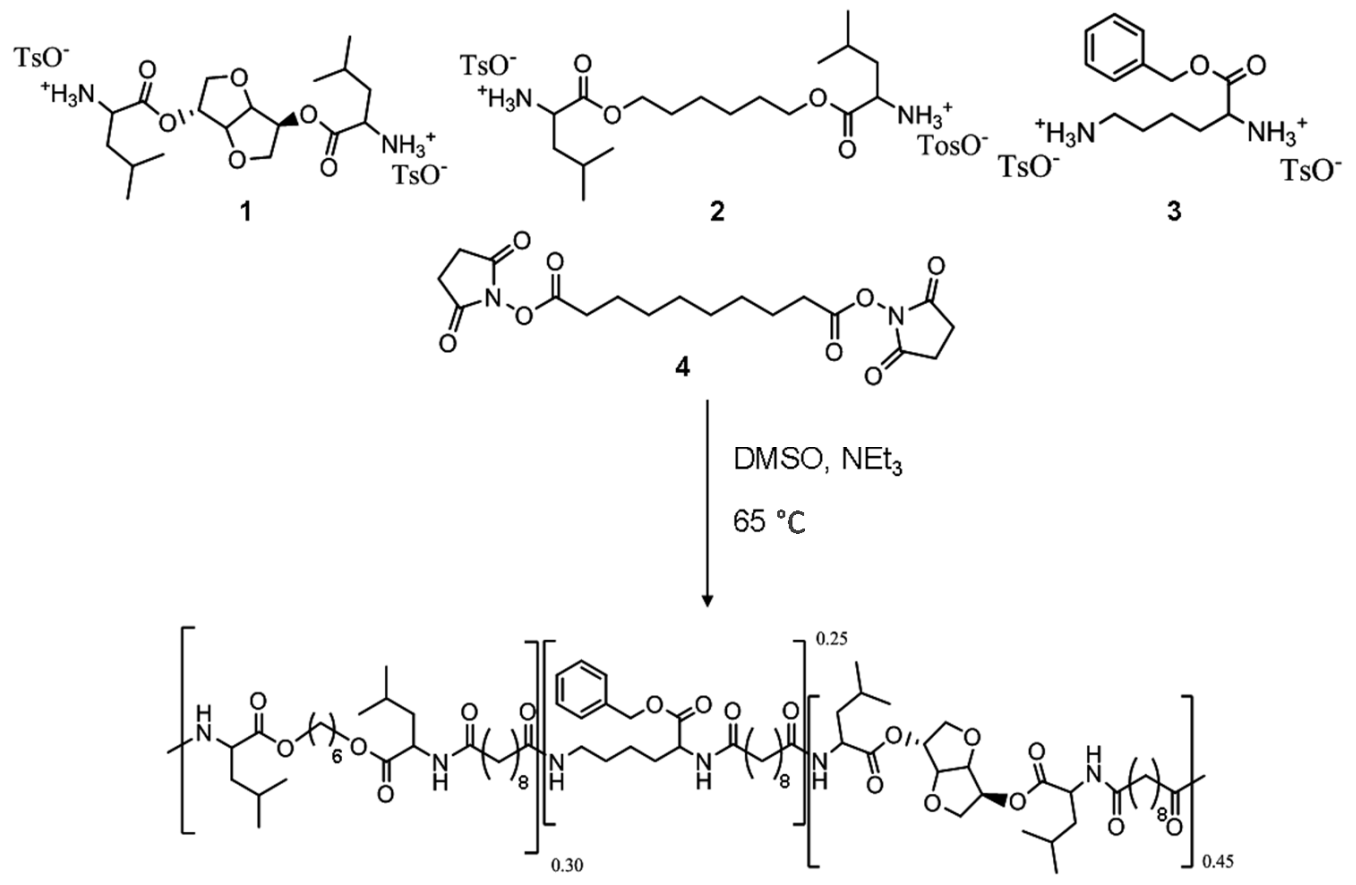


Table 1. Content of the amino acid containing building blocks in the final polymer.

\begin{tabular}{ccc}
\hline Monomers & Theoretical content (mol \%) & Content determined by ${ }^{\mathbf{1}} \mathbf{H}$ NMR (mol \%) \\
\hline $\mathbf{1}$ & 45 & 42 \\
$\mathbf{2}$ & 30 & 32 \\
$\mathbf{3}$ & 25 & 26 \\
\hline
\end{tabular}

Figure 2. ${ }^{1} \mathrm{H}$ NMR spectrum of PEA III Ac Bz recorded in deuterated chloroform. The integration of characteristic protons signals at $4.7 \mathrm{ppm}(\mathrm{s}), 4.1-3.9 \mathrm{ppm}(\mathrm{m})$, and 3.0-2.9 ppm (m) has been used to determine the relative molar ratio of the amino acid containing building blocks in the polymer chain.
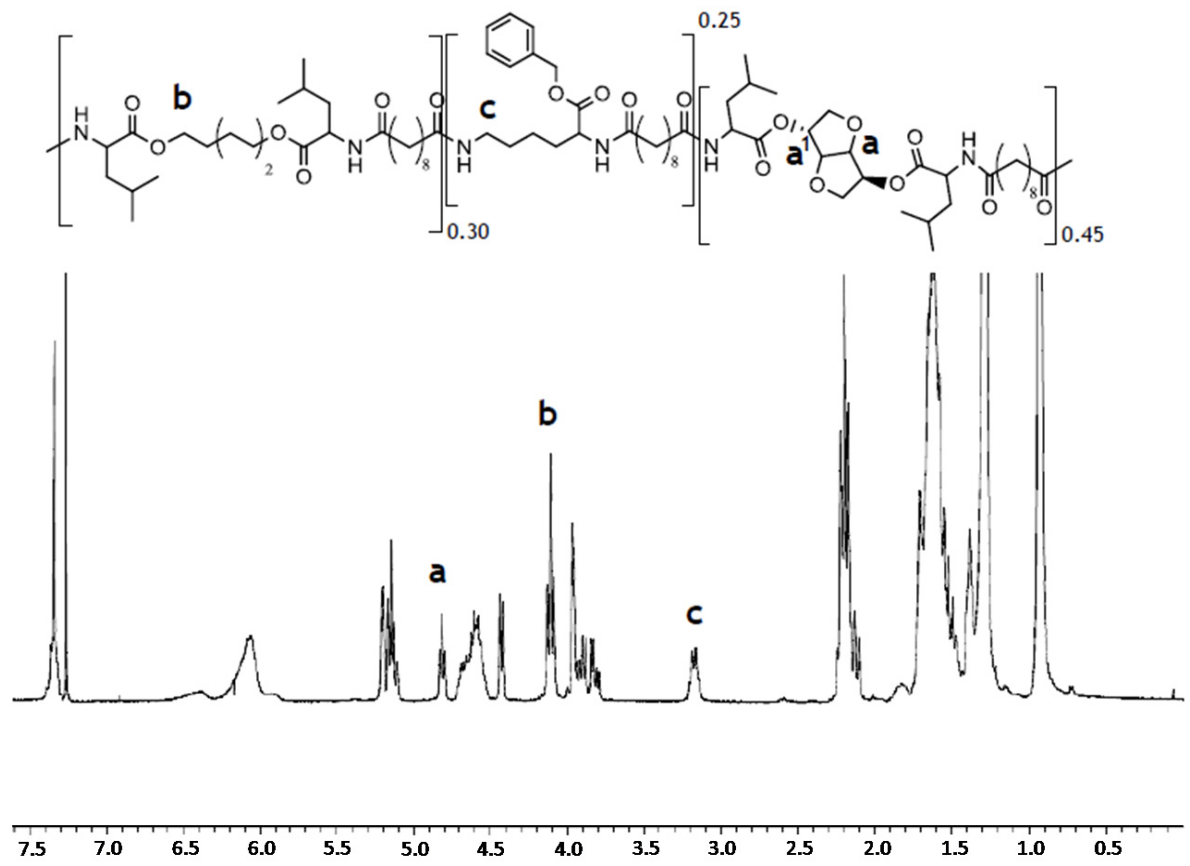

The cryomilled polymer was processed into fibril with a Xcelera mini-extruder (Xcelera, Philadephia, PA, USA) equipped with a DSM (Geleen, The Netherlands) micro fiber spin device for thin fibril spinning. Following 5-10 min residence time at $140{ }^{\circ} \mathrm{C}$ the polymer was stretched into fibrils of $120-300 \mu \mathrm{m}$ in diameter. The extrusion was performed under nitrogen to minimize further oxidative degradation of the polymer. The fibrils were cut into $4 \mathrm{~mm}$ segments and sterilized by gamma irradiation ( $25 \mathrm{kGy}$ ) under cooling conditions by BGS Beta-Gamma-Service GmbH (Wiehl, Germany).

\subsection{Degradation of PEA Fibrils by $\alpha$-Chymotrypsin}

Degradation of the PEA fibrils was analyzed by incubation in a solution of $\alpha$-chymotrypsin $(0.15$ $\mathrm{mg} / \mathrm{mL}, 8.5$ units, type II, lot \#060M7007V) in 0.1 M PBS (phosphate-buffered saline) buffer (pH 7.4) at $37{ }^{\circ} \mathrm{C}$ with gentle shaking $(100 \mathrm{rpm})$. The chymotrypsin solution was replaced every $3-4$ days to maintain a constant level of enzyme activity and to prevent inhibition of the enzyme by degradation products. Sodium azide $(0.05 \mathrm{wt} \%)$ was added to prevent bacterial contamination. After two weeks the surface of the polymer fibrils was analyzed by scanning electron microscopy (SEM). 


\subsection{Degradation of PEA Fibrils by Vitreous Humor in Vitro}

PEA fibrils were incubated in PBS (control), rabbit, human and bovine vitreous humor at $37^{\circ} \mathrm{C}$ in a humidified atmosphere of $95 \%$ air and $5 \% \mathrm{CO}_{2}$. Experiments were performed in triplicate and fibrils were analyzed weekly by fluorescence microscopy. At day one and three, and at four and eight weeks, fibrils were collected and examined by SEM for surface alterations. Vitreous humor was collected from Eye Bank donor eyes after obtaining appropriate informed consent. All experiments involving human tissue were conducted with approval of the institutional ethics committee.

\subsection{Intravitreal and Subconjuctival Implantation and Clinical Follow Up}

Female Chinchilla Bastard Rabbits (Charles River Company, Sulzfeld, Germany) with an average body weight of 2-3 kg were used. All animal experiments were conducted in accordance with the principles for the care and use of research animals and were carried out with the permission and supervision of the Office for the Nature, Environment and Consumer Protection (LANUV), Recklinghausen, Germany. For subconjunctival implantation a radial incision was made into the rabbit conjunctiva and a chamber was prepared by dissecting the conjunctiva from the sclera. One or two dry fibrils were placed into the chamber and the incision was closed with one Vicryl ${ }^{\mathrm{TM}}$ 9-0 suture (Ethicon, Somerville, MA, USA). The implants were observed weekly during a follow up of 1, 2, 4 or 8 weeks.

For intravitreal implantation of dry fibrils, a transscleral paracenthesis was made with a $26 \mathrm{G}$ needle $1.5 \mathrm{~mm}$ below the limbus and a modified $26 \mathrm{G}$ intravenous catheter (BD, Franklin Lakes, NJ, USA) inserted (Figure 3). After removing the catheter needle the PEA fibril was inserted to the catheter with micro forceps and moved forward with the catheter needle into the vitreous humor. The catheter was removed and the intravitreal position of the fibril was documented by video photography (Figure 4). PEA fibrils were explanted after 1, 3 or 5 days and after 2, 4 or 8 weeks. Clinical examinations by funduscopy were done weekly to confirm presence and shape of the fibrils and to observe the status of the fundus.

At specified times eyes were enucleated and after macroscopic observation, the subconjunctival implants and surrounding tissue were subjected to histological analysis. Intravitreal fibrils were explanted, air dried and stored at $4{ }^{\circ} \mathrm{C}$ for SEM examination.

Figure 3. (A) About $1.5 \mathrm{~mm}$ below the limbus a transscleral paranthesis was done and a modified catheter inserted. (B) By pushing forward the fibril with the catheter needle the polymer was implanted into the vitreous.

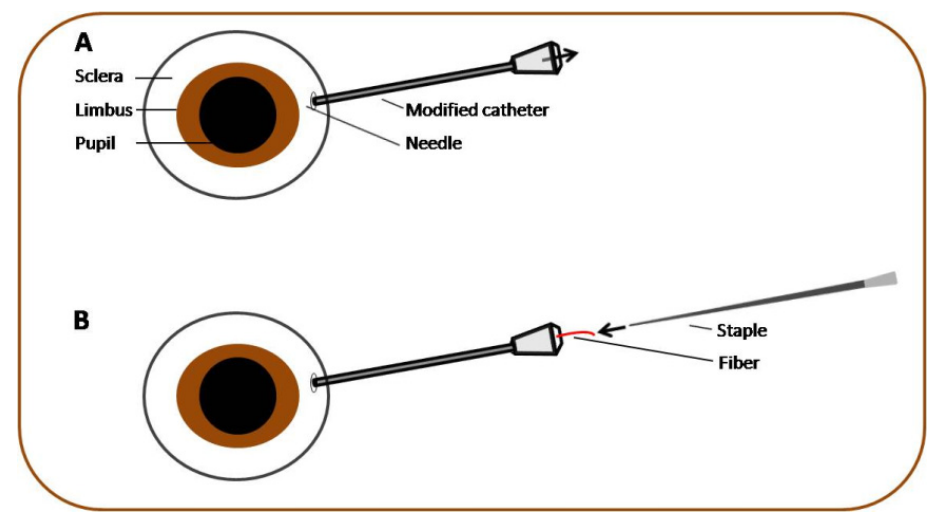


Figure 4. Intravitreal implantation of a dry PEA fibril in rabbits. Note that the fibril (arrow) is still in contact with the catheter protruding into the vitreous humor.

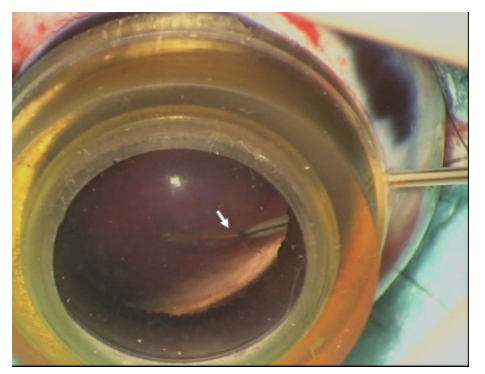

\subsection{Histology}

The subconjunctival PEA implants and surrounding tissue were fixed in $3.7 \%$ formalin and embedded in paraffin. Five micrometer thick sections were stained with H\&E (hematoxylin and eosin) and analyzed for signs of inflammation and tissue reactions. Retinae from eyes with intravitreal implants were fixed in $3.7 \%$ formalin, embedded in paraffin, sectioned, stained with $\mathrm{H} \& \mathrm{E}$ and examined microscopically to evaluate the influence of PEA fibrils on retinal morphology and integrity.

\subsection{Statistics}

All experiments were done at least in triplicate and means \pm SEM were calculated. To compare two parameters in repeated measurements of three groups, the two-way ANOVA (Analysis of Variance) with the Bonferroni correction was performed using the GraphPad Prism 5 (GraphPad Inc., San Diego, CA, USA) software.

\section{Results and Discussion}

Biomaterials are used in several clinical applications, e.g., joint replacement by metal-based materials [24], heart valves [25], blood vessel prosthesis [26,27], breast implants [28], as well as for drug delivery, such as delivery of subcutaneous contraceptives [29] among others. In ophthalmology biomaterials are used extensively as intraocular lenses and contact lenses and since 1996 with the approval by the FDA of Vitrosert ${ }^{\circledR}$ (Bausch \& Lomb), an intravitreal implantable biomaterial, has been used to deliver ganciclovir for the treatment of CMV retinitis in patients with acquired immunodeficiency syndrome (AIDS). Since 1996 a number of biomaterial devices have been developed for intravitreal implantation and are currently in use, such as Ozurdex ${ }^{\circledR}$ (Allergan, Inc., Irvine, CA, USA) [30], Surodex (Allergan, Inc., Irvine, CA, USA) [31,32], Retisert ${ }^{\circledR}$ (Bausch \& Lomb, Inc., Rochester, NY, USA) [33] or Iluvien ${ }^{\circledR}$ (Alimera Sciences, Alpharetta, GA, USA) [34]. However, many of these biomaterials have significant limitations. Non-biodegradable materials for drug delivery devices are large, multicomponent, require surgeries for implantation and must be surgically removed when depleted. Biodegradable materials such as Ozurdex ${ }^{\circledR}$, which can be injected into the vitreous humor, release the drug irregularly. Additionally, the degradation products cause transient acidification of the vitreous humor and are not compatible with many drug species, such as biological therapeutics $[1,12,15]$.

Ideally, a biomaterial for drug delivery should release the drug in a sustained, zero order kinetics, should be compatible with a large number of drugs and biological therapeutic products; the 
degradation products of the biomaterial should not cause any alterations at the implant site or adjacent tissues, and for ophthalmic use the drug-loaded biomaterial should be injectable. PEAs appear to have many of these characteristics, e.g., are capable of sustained drug release and degrade enzymatically by surface erosion [21] and in addition, can be functionalized to meet a wide spectrum of purposes.

\subsection{Effect of Chymotrypsin on Fibril Surface Morphology}

We have treated PEA fibrils with chymotrypsin and shown by SEM that the surface of the fibrils eroded while leaving the core of the fibril intact in agreement with earlier studies [22]. Although chymotrypsin is a digestive system enzyme, it degrades proteins hydrolytically in a manner similar to enzymes present in ocular tissues including the vitreous humor [17,35]. Proteomic analyses has confirmed the presence of a number of enzymes in the vitreous humor such as carbonic anhydrase, the glutathione peroxidase or the prostaglandin D2 synthase [36], and 51 proteases, some of which show increases in diseases like the diabetic retinopathy [37-40]. Contrary to the degradation of PLGA [1], which takes place in the bulk via cleavage of its backbone ester linkages into oligomers and finally monomers [13], degradation of PEA fibrils appears to take place by surface erosion, suggesting that barrier properties of PEA matrix will be preserved during the entire life-time of the implant [22], a characteristic that would allow PEAs to be used for the fabrication of long-term, controlled release devices.

After two weeks of incubation in chymotrypsin the fibrils appear uneven with rough surface and significant patterns of erosion (Figure 5A) whereas the surface of fibrils incubated in PBS is smooth with no sign of degradation (Figure $5 \mathrm{~B}$ ). Both in chymotrypsin and PBS gross examination revealed that the fibrils were considerably shorter in length and increasing in diameter (see below).

Figure 5. (A) After two weeks exposure to $\alpha$-chymotrypsin, there has been obvious erosion of the PEA fibril, as evidenced by the frayed appearance of the fibril surface in SEM micrographs. (B) No evidence of any change was noted in fibrils incubated in PBS.

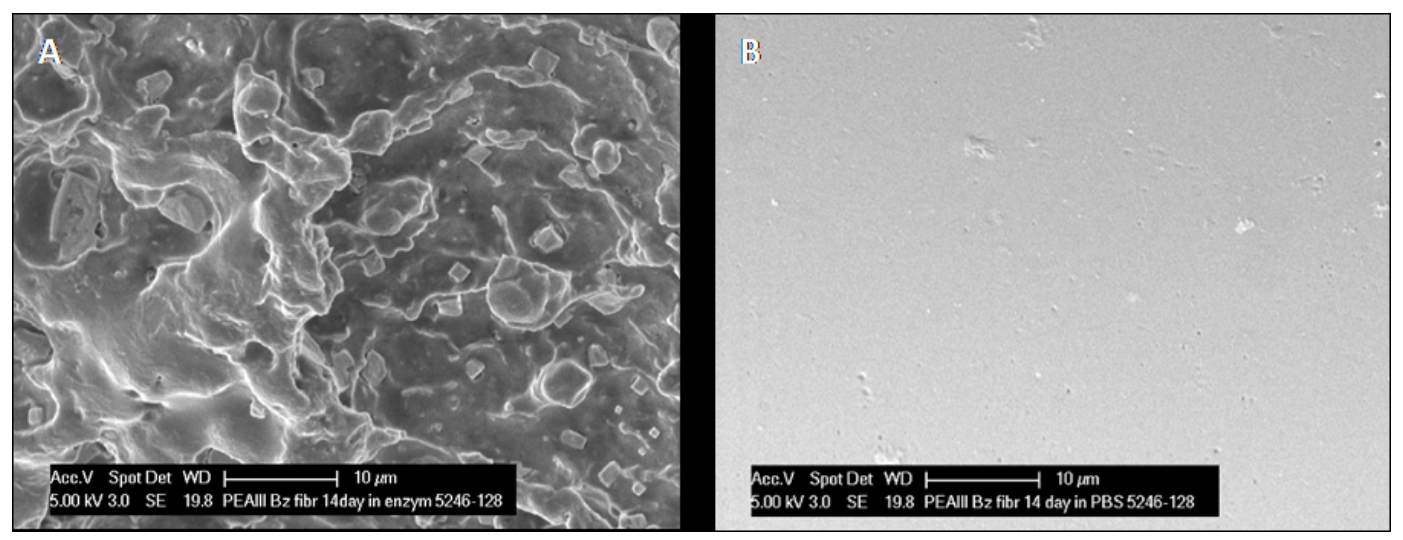

\subsection{Effect of Vitreous Humor on Fibril Morphology}

Incubation of PEA fibrils for eight weeks in rabbit vitreous humor (Figure 6) resulted in a mottled surface suggesting surface erosion, whereas in human vitreous humor (Figure 7) only rounding of the cut edges of the fibrils suggesting shape changes. The differential effect of rabbit and human vitreous humor on the structure of PEA fibrils may result from differences in enzymatic content between human and rabbit vitreous humor. 
Figure 6. (A-C) In PBS, fibrils show rounding and smoothing of the edges after an initial shortening; (D-F) whereas the fibrils incubated in rabbit vitreous humor (rbVitreous) after the initial shortening, show erosive changes, as evidenced by the mottled appearance of the fibrils. Scale bar, $500 \mu \mathrm{m}$. Magnification, $50 \times$.



Figure 7. (A-C) Morphology of PEA fibrils after incubation in human vitreous humor. As in rabbit vitreous humor, fibrils incubated in human vitreous humor contract, but at eight weeks show less erosion changes. Scale bar, $500 \mu \mathrm{m}$. Magnification, $50 \times$.

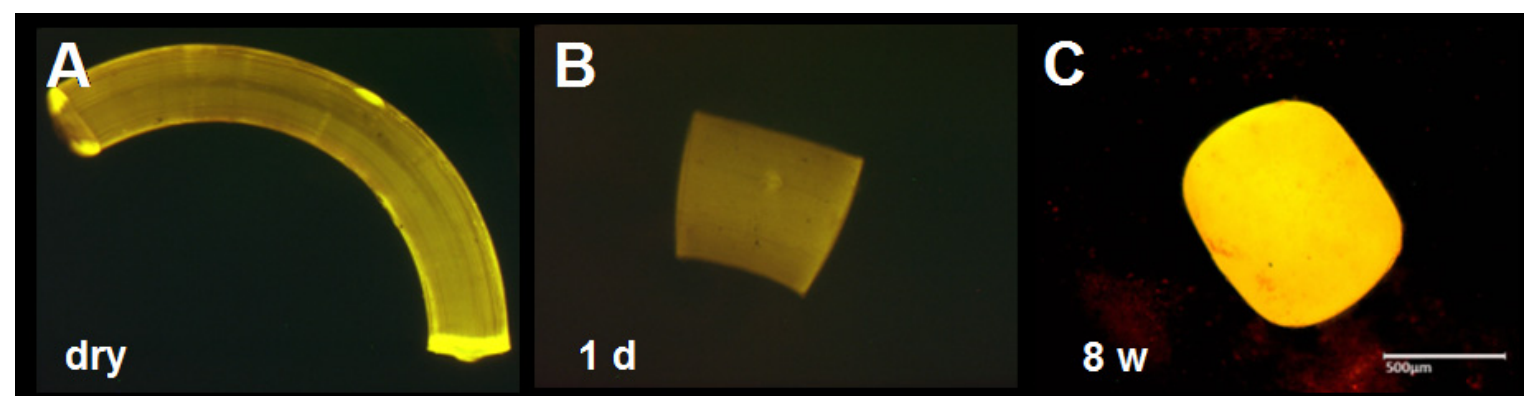

By eight weeks of incubation in rabbit vitreous humor, the surface appeared mottled covering the surface, suggesting erosion of the fibril structure (Figure 6F). However, in human vitreous humor the surface seemed unaltered (Figure 7C).

\subsection{Remodeling of Fibrils in Aqueous Environments}

Subjection of PEA fibrils to aqueous solutions such as PBS, as well as vitreous humor, resulted in distinct remodeling within $24 \mathrm{~h}$ (Figures 6-8). The PEA fibrils keep their shapes, obtained by extrusion, at dry stage since the glass transition temperature $(\mathrm{Tg})$ of the polymer is well above the ambient temperature (room temperature of about $24{ }^{\circ} \mathrm{C}$ ). Once, however, exposed to aqueous environment the polymeric material gets plasticized by water, glass transition temperature drops below 
the temperature of the experiment $\left(37^{\circ} \mathrm{C}\right)$ and fibers start to remodel driven by the tension introduced via extrusion process. The remodeling is relatively quick within $24 \mathrm{~h}$, following the fibril exposure to water and occurs in both vitreous fluid and PBS. The event is not related to degradation since the fibril shows neither weight loss nor changes in the molecular weight of the polymer.

Figure 8. (A) Fibrils incubated in bovine vitreous humor, in rabbit vitreous humor, or in PBS, shorten by $70 \%$ (from 4.03 to $1.19 \mathrm{~mm}$ ) and (B) increase in diameter by $81 \%$ (from 173 to $311 \mu \mathrm{m}$ ) within the first $2 \mathrm{~h}$. During the following $22 \mathrm{~h}$ the fibrils continue to shorten but to a much lesser extent (7.3\%) and reach minimal length and maximal diameter by $24 \mathrm{~h}$. No significant difference in remodeling time and manner among bovine vitreous humor, rabbit vitreous humor or PBS (two-way ANOVA). Data is displayed as mean $\pm \operatorname{SEM}(n=3)$.

A

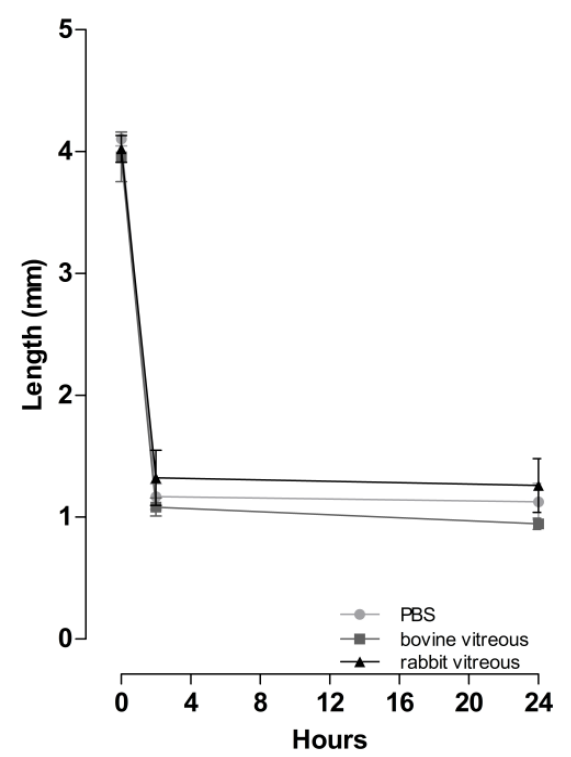

B

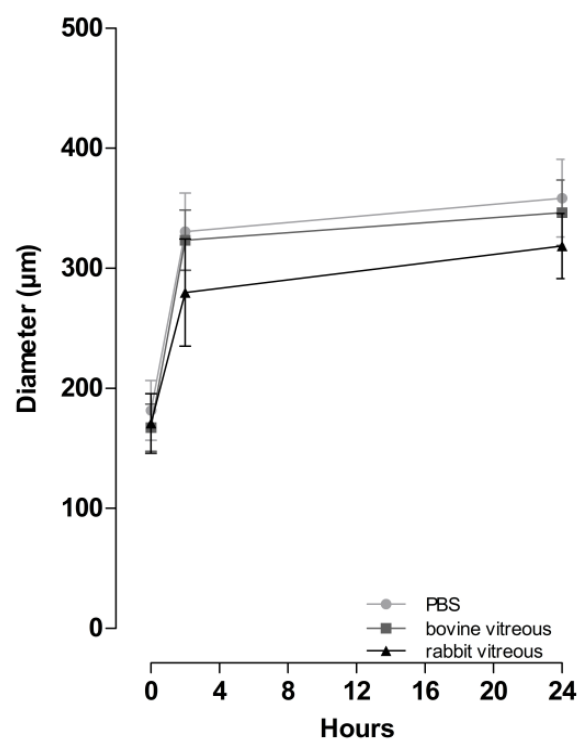

This change in shape of the fibrils in vitreous humor and PBS suggests that PEA fibrils would be useful as drug delivery devices, because drug loaded fibrils could be injected into the vitreous humor where they would remodel, allowing a lower surface to volume ratio (Table 2) thus decreasing adverse side effects related to implant size. Whether drug-loaded fibrils would remodel in a similar fashion without releasing the drug will need to be evaluated.

Table 2. The surface/volume ratio of the PEA fibrils decreased from 22-24 before incubation to 13-16 after $2 \mathrm{~h}$ of incubation in PBS or vitreous humor.

\begin{tabular}{ccc}
\hline Liquid & Incubation time & Surface / volume ratio \\
\hline PBS & $0 \mathrm{~h}$ & 22.7 \\
& $2 \mathrm{~h}$ & 13.8 \\
Rabbit vitreous humor & $0 \mathrm{~h}$ & 24.0 \\
\multirow{3}{*}{ Bovine vitreous humor } & $2 \mathrm{~h}$ & 15.8 \\
& $0 \mathrm{~h}$ & 24.0 \\
& $2 \mathrm{~h}$ & 14.4 \\
\hline
\end{tabular}


In detail, in PBS, rabbit, human (Figures $6 \mathrm{~A}-\mathrm{F}, 7 \mathrm{~A}-\mathrm{C}$ and $8 \mathrm{~A}, \mathrm{~B}$ ) and bovine vitreous humor (Figure 8A,B) the fibrils become considerably shorter and thicker within $24 \mathrm{~h}$ with no evident surface alterations. To quantify the change in shape in PBS, rabbit and bovine vitreous humor the length and the diameter of the fibrils $(n=3)$ was measured before and after $2 \mathrm{~h}$ and $24 \mathrm{~h}$ of incubation (Figure 9). The fibrils were photographed, and length and diameter determined with the Diskus imaging software (Hilgers, Germany).

Figure 9. (A-C) Follow-up of PEA fibrils implanted in the rabbit conjunctiva. During the eight weeks of the experiment, no abnormalities and only few signs of inflammatory reactions were observed at the site of implantation. Note the clear conjunctiva with the fibril clearly visible (arrows). (D) The inset shows a normal implantation site of an enucleated eye with the fibril (arrow) clearly visible eight weeks after implantation.

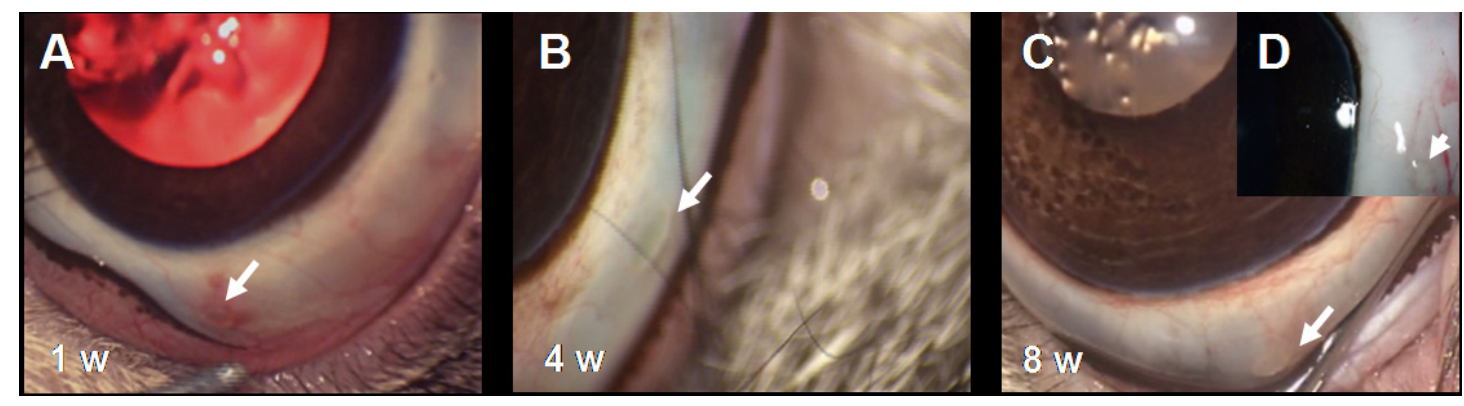

During the first $2 \mathrm{~h}$ the fibrils decreased in length from 4.10 to $1.17 \mathrm{~mm}$ (or by $72 \%$ ) in PBS, from 4.02 to $1.32 \mathrm{~mm}$ (or by 67\%) in rabbit vitreous humor and from 3.96 to $1.08 \mathrm{~mm}$ (or by $72 \%$ ) in bovine vitreous humor. During the first $2 \mathrm{~h}$ the fibrils also increased in diameter from 181.7 to $330.7 \mu \mathrm{m}$ (or by $85 \%$ ) in PBS, from 170.8 to $279.7 \mu \mathrm{m}$ (or by $63 \%$ ) in rabbit vitreous humor and from 167.2 to $323.3 \mu \mathrm{m}$ (or by $95 \%$ ) in bovine vitreous humor. During the next $22 \mathrm{~h}$ of measurements the fibrils decreased in length by only 5\% in PBS, by $5 \%$ in rabbit vitreous humor and by $12 \%$ in bovine vitreous humor and increased in diameter by $9 \%$ in PBS, by $18 \%$ in rabbit vitreous humor and by $7 \%$ in bovine vitreous humor (Figure 8).

\subsection{Biocompatibility of PEA in Conjunctival Tissue}

PEAs have been proposed as drug delivery vehicles and in vitro studies have shown that PEAs are not toxic $[41,42]$ and, as we report here, become degraded by surface erosion. However, it is not known how these polymers behave in vivo in relation to toxicity, immunological response, degradation and morphological architecture. To define the effects of the ocular environment PEA fibrils were implanted in the vitreous humor and subconjunctivally in rabbits.

No inflammatory reactions were observed by direct examination or by video documentation of the fibrils implanted subconjunctivally during the eight weeks of the experiments. Shown in Figure 9 are photographs captured from the videos at 1, 4 and 8 weeks; note that at one week (Figure 9A) in this particular implant a mild vascular reaction is evident, which is especially pronounced at the site of the suture and not in the immediate vicinity of the implanted fibril (arrow). A similar mild inflammatory response was observed in one third of the implants, which in all cases resolved by the fourth week. Figure 9B-D show an implant at 4 and 8 weeks after implantation; note that there are no signs of 
inflammation; the tissue surrounding the PEA fibril is free of new vessels, edema or other indications for inflammation. Similar to our in vitro data, PEA fibrils showed considerable reshaping after subconjunctival implantation; within $24 \mathrm{~h}$ the fibrils become considerably shorter and thicker.

\subsection{Histology of Fibrils and Subconjunctival Implantation Site}

After enucleation, $84 \%$ of the subconjunctival PEA implants were located, explanted with surrounding tissue, and processed for histology. H\&E staining showed that by week one a thin capsule had formed around the implanted fibrils (Figure 10) which persisted throughout the eight weeks of the experiment. Cells at the capsule edge were fibroblasts, with granulation tissue evident in some specimens. Along the capsule and adjacent to the implant site, lymphocytes and macrophages were observed (arrows) indicating a mild, normal tissue reaction to a foreign material and at week one a single plasma cell was observed at the implant site. However the total number of inflammatory cells remained low for the eight weeks of follow-up indicating only a slight immunological reaction. No giant cells or eosinophils were observed in the vicinity of the implant. The thin capsule clearly demonstrates the excellent biocompatibility of PEA polymers, but raises the question of permeability of the drug released by the PEA fibrils through the capsule. Studies of drug concentration in the tissue adjacent to implanted drug loaded microfibrils will be carried out to answer this question.

Figure 10. (A) Histology of the subconjunctival implant site. By week one after the implantation of the PEA fibrils, a thin capsule (asterisk) had enveloped the fibril; $(\mathbf{B}, \mathbf{C})$ the capsule persisted for the eight weeks of the experiment. Fibroblasts were consistently observed adjacent to the capsule. Rare leukocytes are visible in the magnified sections (examples are marked with arrows). Scale bar, $200 \mu \mathrm{m}$. Magnification, 200×.

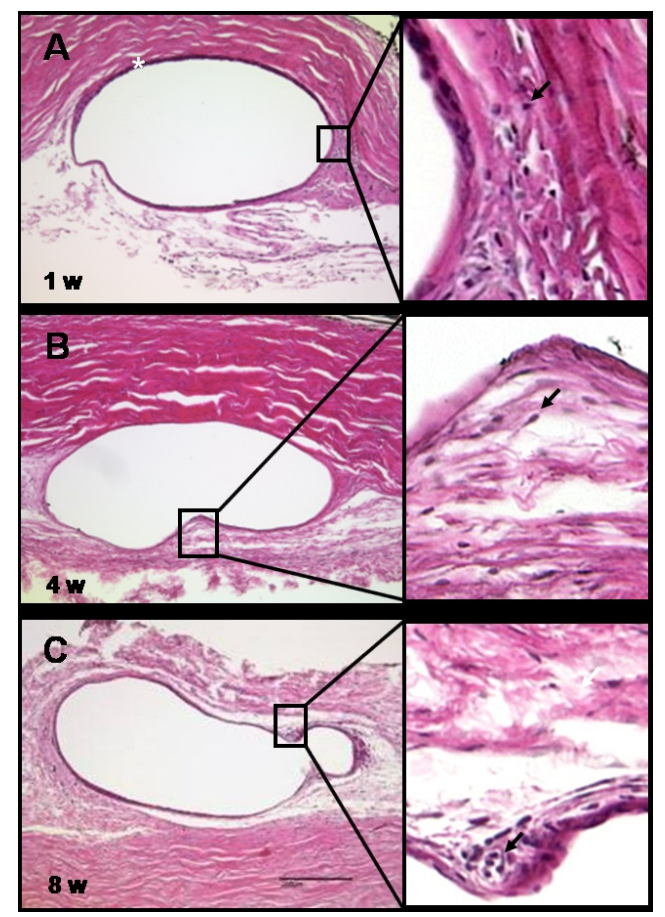

Summarized, histological analysis of the fibrils and subconjunctival implantation site showed that the fibrils became encapsulated by a thin capsule that was surrounded by fibroblasts and only a few 
immune competent cells indicating a mild tissue reaction to a foreign material. No granulocytes were determined, recognizable by granulae and segmented nuclei. Furthermore no multinucleated giant cells were detected. Only some areas with small accumulations of lymphocytes and macrophages, discernible by their round shape and big nuclei, could be identified. No cells were observed in direct contact or attached to the fibrils indicating that this particular PEA will not support cell attachment or growth and therefore the polymer keeps good promise in variety of medical applications where cell attachment or growth is not desirable.

\subsection{Fundus and Retinal Morphology after Intravitreal Implantation of PEA Microfibrils}

Implantation into the vitreous humor was accomplished by injecting the fibrils through a $26 \mathrm{G}$ needle transsclerally and as expected the procedure was simple and uneventful. Fibrils implanted in the vitreous humor remained within the vitreous humor body throughout the eight weeks of the experiment (Figures 11 and 12); similar to the subconjunctival implants, the intravitreally implanted fibrils became shorter and thicker. Fundus photographs at 1, 4 and 8 weeks show a normal eye without any evidence of damage, inflammation or retinal detachment (Figure 11A-C). After enucleation the eyes were macroscopically evaluated and photographed. Figure 12A,B are photographs of the fibrils in an enucleated eye at 4 and 8 weeks, respectively; note that the retina remained attached, that blood vessels and myelinated nerve fibrils (arrows) display no abnormalities. Gross observation of the fibrils did not show fast material erosion during the 8 weeks of the experiment. These results confirm the expected slow polymer degradation, preferable for long-term ocular drug delivery. Further research is necessary to determine long term degradation kinetics in vivo.

Figure 11. (A) Fundus examination of eyes with PEA fibrils implanted intravitreally. At one week the fibrils are clearly visible directly behind the lens. (B) After four weeks the fibril is still suspended within the vitreous humor. The fundus, the papilla (black arrow), and the nerve (red arrow) appear normal. (C) At eight weeks after implantation the PEA fibrils (white arrows) are still suspended within the vitreous humor and appear intact by gross examination.

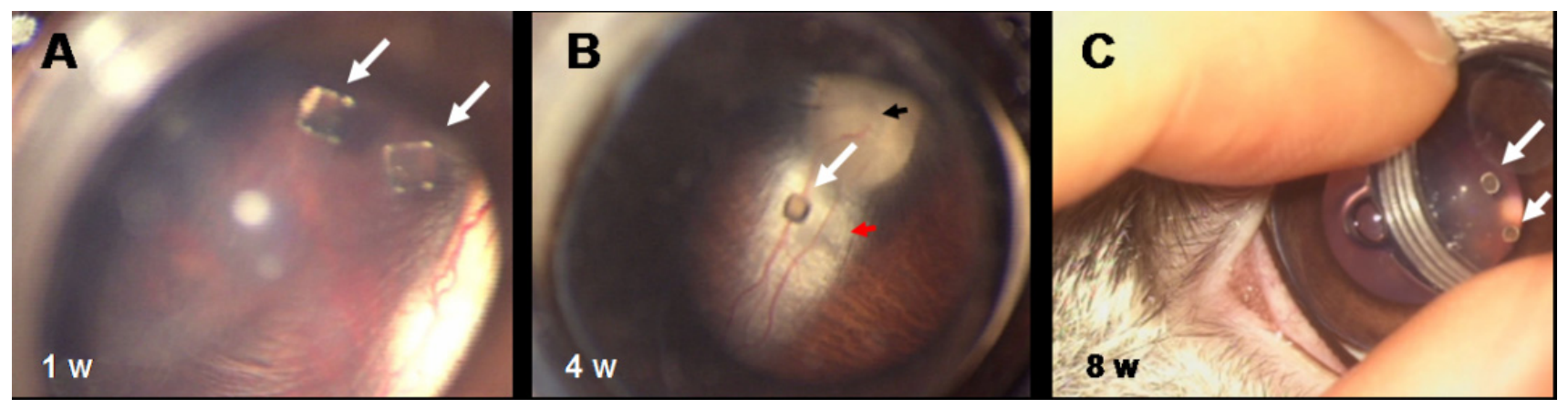


Figure 12. Macroscopic examination of enucleated eyes with intravitreal implants of PEA fibrils. Examination of enucleated eyes with intravitreal PEA fibril implants (white arrows) (A) four and (B) eight weeks after implantation shows an attached retina with papilla (black arrow) and nerve (red arrow) perfused and myelinated.

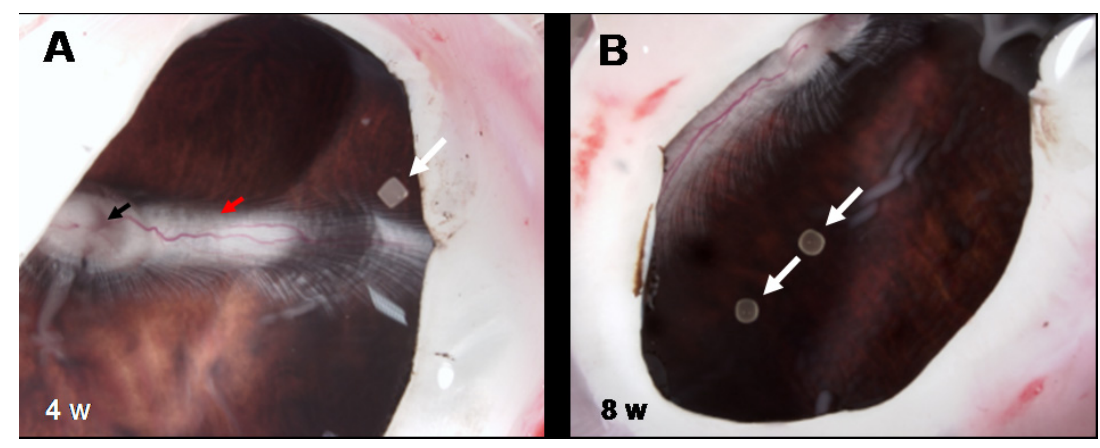

Histologically the retinae of eyes with intravitreal implants were normal. No evidence of any inflammatory response or any alterations of the retinal architecture was apparent at 1, 4 or 8 weeks post-implantation (Figure $13 \mathrm{~A}-\mathrm{C}$ ). This indicates that if any degradation of the implanted fibrils had taken place, the degradation products did not damage or alter the retina (Figure 13); note the integrity of the photoreceptor layer ("P”) and ganglion cell layer (“G”).

Figure 13. Histology of the retina from eyes implanted with PEA fibrils intravitreally. At (A) one, (B) four, and (C) eight weeks after intravitreal implantation the retina is normal with no evidence of any alteration. Note a normal and intact photoreceptor layer ("P") and ganglion cell layer (“G”). Scale bar, $100 \mu \mathrm{m}$. Magnification, 200×.

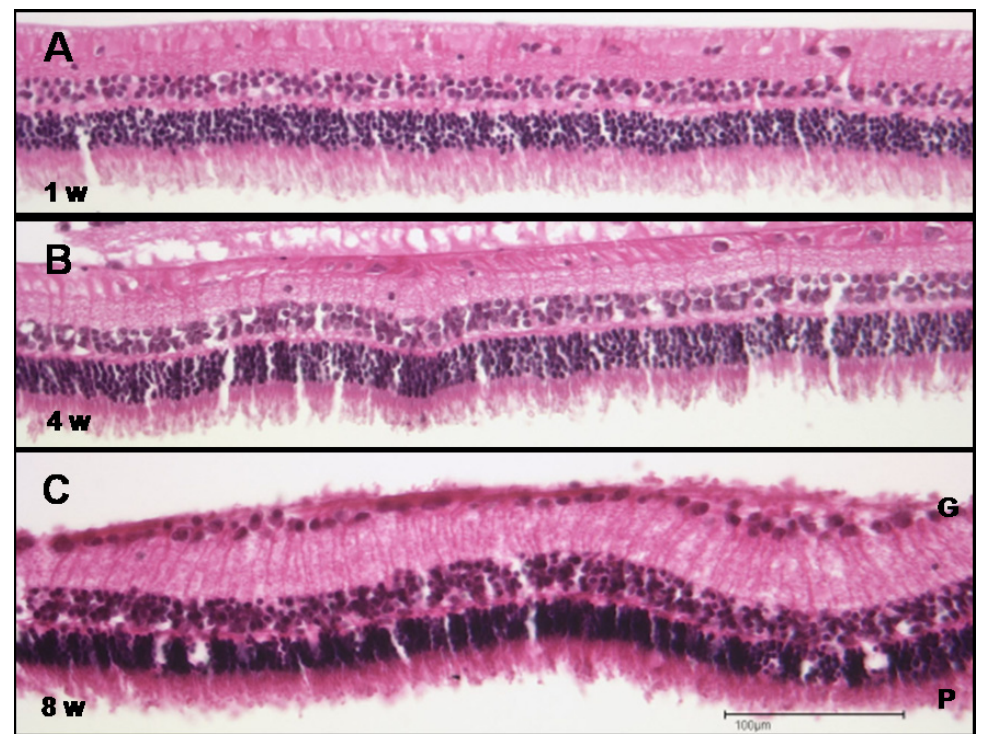

\section{Conclusions}

Herein, we show that PEA is a biocompatible and biodegradable polymer. In vitro and in vivo analyses unveiled the promising properties of PEA polymers for ophthalmic purposes. SEM of PEA fibrils incubated with chymotrypsin confirmed the previous reports that fibrils degrade via surface erosion mechanism enabling new approaches towards development of vehicles for sustained and 
controlled drug release. The change of shape in vitreous humor would offer an additional advantage for implantation in ocular tissues such as diminished side effects and discomfort related to implant size. In vivo implantation showed excellent tissue biocompatibility and tolerance in rabbit eyes. Future work will analyze the pharmacokinetics of drug release from drug loaded PEA microfibrils. Taken together, the results presented here indicate that PEA fibrils are promising polymers for controlled long term drug release in the ocular environment.

\section{Acknowledgments}

This work was supported by DSM. We like to thank the Interdisziplinäres Zentrum für Klinische Forschung (IZKF) Aachen, University Hospital Rheinisch-Westfälische Technische Hochschule (RWTH) Aachen for the use of their facilities for histology and microscopy. The authors express their thanks to Claudia Etzkorn (Clinic of Ophthalmology, University Hospital RWTH Aachen) and Sabine Diarra (Department of Vitreoretinal Surgery, University Hospitals of Geneva) for their support and collaboration in the animal studies.

\section{Conflicts of Interest}

This study was financially supported by DSM, Geleen.

\section{References}

1. Lee, S.S.; Hughes, P.; Ross, A.D.; Robinson, M.R. Biodegradable implants for sustained drug release in the eye. Pharm. Res. 2010, 27, 2043-2053.

2. Yasukawa, T.; Tabata, Y.; Kimura, H.; Ogura, Y. Recent advances in intraocular drug delivery systems. Recent Pat. Drug Deliv. Formul. 2011, 5, 1-10.

3. Adelman, R.A.; Zheng, Q.; Mayer, H.R. Persistent ocular hypertension following intravitreal bevacizumab and ranibizumab injections. J. Ocul. Pharm. Ther. 2010, 26, 105-110.

4. Krishnan, R.; Goverdhan, S.; Lochhead, J. Submacular haemorrhage after intravitreal bevacizumab compared with intravitreal ranibizumab in large occult choroidal neovascularization. Clin. Exper. Ophthalmol. 2009, 37, 384-388.

5. Baeteman, C.; Hoffart, L.; Galland, F.; Ridings, B.; Conrath, J. Hématomes maculaires après injections intravitréenes d'anti-VEGF pour dégégenerescence maculaire liée à l'age: Sèrie rétrospective. J. Fr. Ophthalmol. 2009, 32, 309-313. (in French)

6. Martin, D.F.; Maguire. M.G.; Ying, G.S.; Grunwald, J.E.; Fine, S.L.; Jaffe, G.J. Ranibizumab and bevacizumab for neovascular age-related macular degeneration. New Engl. J. Med. 2011, 364, 1897-1908.

7. Short, B.G. Safety evaluation of ocular drug delivery formulations: Techniques and practical considerations. Toxicol. Pathol. 2008, 36, 49-62.

8. Mohammad, D.A.; Sweet, B.V.; Elner, S.G. Retisert: Is the new advance in treatment of uveitis a good one? Ann. Pharmacother. 2007, 41, 449-455.

9. Kompella, U.B.; Kadam, R.S.; Lee, V.H.L. Recent advances in ophthalmic drug delivery. Ther. Del. 2010, 1, 435-456. 
10. Hebson, C.B.; Srivastava, S.K. A functional, nonfunctioning retisert implant. Ocul Immunol Inflamm 2011, 19, 210-211.

11. Herrero-Vanrell, R.; Refojo, M.F. Biodegradable microshperes for vitreoretinal drug delivery. $A d v$. Drug Del. Rev. 2001, 52, 5-16.

12. London, N.J.S.; Chiang, A.; Haller, J.A. The dexamethasone drug delivery system: Indications and evidence. Adv. Therapy. 2011, 28, 351-366.

13. Makadia, H.K.; Siegel, S.J. Poly lactic-co-glycolic acid (PLGA) as biodegradable controlled Drug delivery carrier. Polymers 2011, 3, 1377-1397.

14. Liu, Y.; Ghassemi, A.H.; Hennink, W.E.; Schwendemann, S.P. The microclimate Ph in Poly(D,L-lactide-co-hydroxymethyl glycolide) microspheres during biodegradation. Biomaterials 2012, 33, 7584-7593.

15. Van de Weert, M.; Hennink, W.E.; Jiskoot, W. Protein instability in poly(lactic-co-glycolic acid) microparticles. Pharm. Res. 2000, 17, 1159-1167.

16. Mihov, G.; Draaisma, G.; Dias, A.A.; Turnell, B.; Gomurashvili, Z. Degradable polyesteramides: A novel platform for sustained drug delivery. J. Control. Release 2010, 148, 46-47.

17. Guo, K.; Chu, C.C. Biodegradable and injectable paclitaxel-loaded poly(ester amide)s microspheres: Fabrication and characterization. J. Biomed. Mater. Res. 2009, 89, 491-500.

18. Lee, S.H.; Szinai, I.; Carpenter, K.; Katsarava, R.; Jokhadze, G.; Chu, C.C.; Huang, Y. Verbeken, E.; Bramwell, O.; de Scheerder, I.; et al. In-vivo biocompatibility evaluation of stents coated with a new biodegradable elastomeric and functional polymer. Coron. Artery Dis. 2002, 13, 237-241.

19. DeFife, K.M.; Grako, K.; Cruz-Aranda, G.; Price, S.; Chantung, R.; Macpherson, K.; Khoshabeh, R.; Gopalan, S.; Turnell, W.G. Poly(ester amide) co-polymers promote blood and tissue compatibility. J. Biomater. Sci. 2009, 20, 1495-1511.

20. Sun, H.; Meng, F.; Dias, A.A.; Hendriks, M.; Feijen, J.; Zhong, Z. $\alpha$-Amino acid containing degradable polymers as functional biomaterials: Rational design, synthetic pathway, and biomedical applications. Biomacromolecules 2011, 12, 1937-1955.

21. Ghaffar, A.; Draaisma, G.J.J.; Mihov, G.; Dias, A.A.; Schoenmakers, P.J.; van der Wal, S. Monitoring the in vitro enyzme-mediated degradation of degradable poly(ester amide) for controlled drug delivery by Lc-Tof-Ms. Biomacromolecules 2011, 12, 3243-3251.

22. Tsitlanadze, G.; Machaidze, M.; Kviria, T.; Djavakhishvili, N.; Chu, C.C.; Katsarava, R. Biodegradation of amino-acid-based poly(ester amide)s: In vitro weight loss and preliminary in vivo studies. J. Biomater. Sci Polym. Ed. 2004, 15, 1-24.

23. Katsarava, R.; Beridze, V.; Arabuli, N.; Kharadze, D.; Chu, C.C.; Won, C.Y. Amino acid-based bioanalogous polymers. Synthesis, and study of regular poly(ester amide)s based on bis( $\alpha$-amino acid) $\alpha, \omega$-alkylene diesters, and aliphatic dicarboxylic acids. J. Polym. Sci. A Polym. Chem. 1999, 37, 391-407.

24. Bozic, K.; Browne, J.; Dangles, C.J.; Manner, P.A.; Yates, A.J., Jr.; Weber, K.L.; Boyer, K.M.; Zemaitis, P.; Woznica, A.; Turkelson, C.M.; et al. Modern metal-on-metal hip implants. J. Am. Acad. Orthop. Sur. 2012, 20, 402-406.

25. Lam, M.T.; Wu, J.C. Biomaterial applications in cardiovascular tissue repair and regeneration. Expert. Rev. Cardiovasc. Ther. 2012, 10, 1039-1049. 
26. Akhmedov, S.; Afanas'ev, S.A.; Egorova, M.V.; Ivanov, A.V.; Rogovskaia, Y.V.; Usov, V.Y.; Shvedov, A.N.; Steinhoff, G. Cell-free collagen-based scaffolds used for making blood vessels in cardiovascular surgery. Angiol. Sosud. Khir. 2012, 18, 7-12.

27. Murphy, S.V.; Atala, A. Organ engineering - Combining stem cells, biomaterials, and bioreactors to produce bioengineered organs for transplantation. Bioessays 2012, 35, 163-173.

28. Puskas, J.E.; Luebbers, M.T. Breast implants: The good, the bad and the ugly. Can nanotechnology improve implants? Wiley Interdiscip. Rev. Nanomed. Nanobiotechnol. 2012, 4, 153-168.

29. Stoddard, A.; McNicholas, C.; Peipert, J.F. Efficacy and safety of long-acting reversible contraception. Drugs 2011, 71, 969-980.

30. Chan, A.; Leung, L.-S.; Blumenkranz, M.S. Critical appraisal of the clinical utility of the dexamethasone intravitreal implant $\left(\right.$ Ozurdex $\left.{ }^{\circledR}\right)$ for the treatment of macular edema related to branch retinal vein occlusion or central retinal vein occlusion. Clin. Ophthalmol. 2011, 5, 1043-1049.

31. Tan, D.T.; Chee, S.-P.; Lim, L.; Theng, J.; van Ede, M. Randomized clinical trial of Surodex steroid drug delivery system for cataract surgery: anterior versus posterior placement of two surodex in the eye. Ophthalmology 2001, 108, 2172-2181.

32. Kodama, M.; Numaga, J.; Yoshida, A.; Kaburaki, T.; Oshika, T.; Fujino, Y.; Wu, G.-S.; Rao, N.A.; Kawashima, H. Effects of a new dexamethasone-delivery system (Surodex) on experimental intraocular inflammation models. Graefe's Arch. Clin. Exp. Ophthalmol. 2003, 241, 927-933.

33. Pavesio, C.; Zierhut, M.; Bairi, K.; Comstock, T.L.; Usner, D.W. Evaluation of an intravitreal fluocinolone acetonide implant versus standard systemic therapy in noninfectious posterior uveitis. Ophthalmology 2010, 117, 567-575.

34. Kane, F.E.; Burdan, J.; Cutino, A.; Green, K. Iluvien ${ }^{\mathrm{TM}}$ : A new sustained delivery technology for posterior eye disease. Expert Opin. Drug Del. 2008, 5, 1039-1046.

35. Jacobson, B. Degradation of glycosaminoglycans by extracts of calf vitreous hyalocytes. Exp. Eye Res. 1984, 39, 373-385.

36. Gao, B.-B.; Clermont, A.; Rook, S.; Fonda, S.J.; Srinivasan, V.J.; Wojtkowski, M.; Fujimoto, J.G.; Avery, R.L.; Arrigg, P.G.; Bursell, S.E.; et al. Extracellular carbonic anhydrase mediates hemorrhagic retinal and cerebral vascular permeability through prekallikrein activation. Nat. Med. 2007, 13, 181-188.

37. Hara, H.; Oh-hashi, K.; Yoneda, S.; Shimazawaa, M.; Inatani, M.; Tanihara, H.; Kiuchi, K. Elevated neprilysin activity in vitreous of patients with proliferative diabetic retinopathy. Mol. Vis. 2006, 12, 977-982.

38. Ishizaki, E.; Takai, S.; Ueki, M.; Maeno, T.; Maruichi, M.; Sugiyama, T.; Oku, H.; Ikeda, T.; Miyazaki, M. Correlation between angiotensin-converting enzyme, vascular endothelial growth factor, and matrix metalloproteinase-9 in the vitreous of eyes with diabetic retinopathy. Am. J. Ophthalmol. 2006, 141, 129-134.

39. Wang, H.; Feng, L.; Hu, J.W.; Xie, C.L.; Wang, F. Characterisation of the vitreous proteome in proliferative diabetic retinopathy. Proteome Sci. 2012, 10, doi:10.1186/1477-5956-10-15.

40. Aretz, S.; Krohne, T.U.; Kammerer, K.; Warnken, U.; Hotz-Wagenblatt, A.; Bergmann, M.; Stanzel, B.V.; Kempf, T.; Holz, F.G.; Schnölzer, M.; et al. In-depth mass spectrometric mapping of the human vitreous proteome. Proteome Sci. 2013, 11, doi:10.1186/1477-5956-11-22. 
41. Knight, D.K.; Stutchbury, R.; Imruck, D.; Halfpap, C.; Lin, S.; Langbein, U.; Gillies, E.R.; Mittler, S.; Mequanint, K. Focal contact formation of vascular smooth muscle cells on Langmuir-Blodgett and solvent-cast films of biodegradable poly(ester amide)s. ACS Appl. Mater. Interfaces 2012, 4, 1303-1312.

42. He, P.; Tang, Z.; Lin, L.; Deng, M.; Pang, X.; Zhuang, X.; Chen, X. Novel biodegradable and $\mathrm{pH}$-sensitive poly(ester amide) microspheres for oral insulin delivery. Macromol. Biosci. 2012, 12, 547-556.

(C) 2014 by the authors; licensee MDPI, Basel, Switzerland. This article is an open access article distributed under the terms and conditions of the Creative Commons Attribution license (http://creativecommons.org/licenses/by/3.0/). 